\title{
Cornelia de Lange syndrome - a rarely seen disorder
}

\author{
Y Çekmez, ${ }^{1} \mathrm{MD}$; N Pişkinpaşa, ${ }^{2} \mathrm{MD} ; \mathrm{T}$ Tos, ${ }^{3} \mathrm{MD}$ \\ ${ }^{1}$ Department of Obstetrics and Gynaecology, Umraniye Medical and Research Hospital, Istanbul, Turkey \\ ${ }^{2}$ Department of Obstetrics and Gynaecology, Manisa Government Hospital, Manisa, Turkey \\ ${ }^{3}$ Department of Obstetrics and Gynaecology, Dr Sami Ulus Medical and Research Hospital, Ankara, Turkey
}

Corresponding author: Y Çekmez (yaseminkandicekmez@hotmail.com)

\begin{abstract}
Cornelia de Lange syndrome (CdLS) is a rare genetic disorder of unknown causation, associated with multiple congenital anomalies. Prenatal genetic diagnosis is possible, and the syndrome can occur in subsequent pregnancies of families with affected children as a result of mosaicism. The syndrome has been diagnosed antenatally by careful ultrasound examination, but is usually only diagnosed after birth. We report the case of a patient admitted to our clinic with intrauterine death of the fetus. CdLS was diagnosed on the basis of multiple structural abnormalities seen after delivery.
\end{abstract}

S Afr J Obstet Gynaecol 2015;21(2):52-53. DOI:10.7196.SAJOG.890

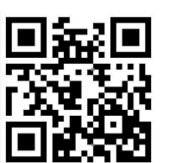

Cornelia de Lange syndrome (CdLS), also known as Brachmann de Lange syndrome, is a rarely seen genetic disorder characterised by facial features such as monobrow (synophrys), long eyelashes, anteverted nostrils, a long filtrum and thin lips. Failure to thrive, mental retardation, hirsutism and multiple congenital anomalies have also been described. ${ }^{[1]}$

The prevalence of CdLS has been reported to vary between $1 / 10000$ and $1 / 50000$ births, and there is a $2-5 \%$ risk of recurrence in a subsequent pregnancy. The causation appears to be multifactorial, and most cases are sporadic. Inheritance is autosomal dominant or recessive. ${ }^{[1,2]}$ On microscopic examination, a decrease in the number of oligodendrocytes and a myelin defect in transverse fibrils are seen. A relationship between total deficiency of pregnancy-associated plasma protein A, a gestational protein, and CdLS has been reported. Increased nuchal translucency and a dysmorphic appearance of the upper extremities can be useful in diagnosing CdLS prenatally. Degrees of severity differ even between members of the same family, and it is impossible to make a diagnosis phenotypically in most cases. We report the case of a patient admitted to our clinic with intrauterine death of the fetus. CdLS was diagnosed on the basis of multiple structural abnormalities seen after delivery.

\section{Case report}

A 27-year-old woman, para 5, gravida 7, was admitted to the Dr Sami Ulus Medical and Research Hospital, Ankara, Turkey, with decreased fetal movement, backache and groin pain. Her last delivery had been by caesarean section, she did not know her date of last menstrual period, there was no consanguinity, and her surviving children had no anomalies. There was no other medical history of note. She had found out that she was pregnant when she saw a doctor at about 8 weeks' gestation, but had not been followed up. TORCH screening at 8 weeks had been negative.

An ultrasound scan showed no fetal heartbeat. The biparietal diameter was $88.3 \mathrm{~mm}$ (corresponding to a gestation of 35 weeks and 5 days) and the femur length $68.1 \mathrm{~mm}$ (35 weeks). Pelvic examination showed cephalic presentation, and the cervix was $4 \mathrm{~cm}$ dilated with $60 \%$ effacement. The patient was in labour, and as she had had a previous caesarean section and requested tubal ligation, emergency caesarean section was performed. A dead fetus weighing $2980 \mathrm{~g}$ and $48 \mathrm{~cm}$ in length was delivered. The fetus had dysmorphic facial features, a depressed nasal bridge, synophrys, a wide frenulum, hirsutism, low-set ears, ectrodactyly in the left hand and phocomelia on right (Figs 1 and 2). The skin was stained with yellowbrown meconium. On the basis of these findings, CdLS was diagnosed and genetic counselling was provided to the family. Autopsy of the fetus was suggested, but the family did not agree to it.

\section{Discussion}

Some signal pathways with important modulation roles in development of the

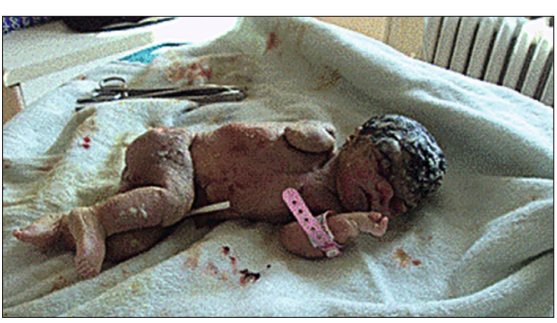

Fig. 1. Typical facial features in CdLS: monobrow (synophrys), long eyelashes, anteverted nostrils, long filtrum and thin lips.

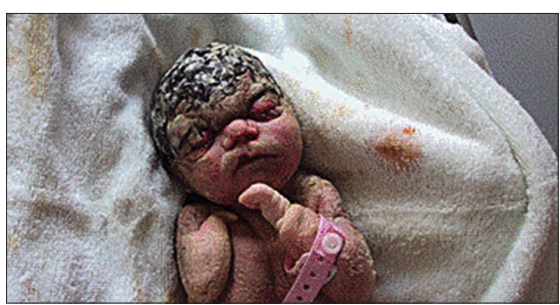

Fig. 2. Ectrodactyly on the left hand and phocomelia on the right.

embryo, starting from the early stages of morphogenesis, also have a basic role in creating the anatomical structure of the musculoskeletal system. Although malformations related to the basic differentiation processes are very rare in humans, most congenital anomalies are related to these processes in the early phases of morphogenesis. This applies especially to disorders that have syndromic characteristics as a result of their anomaly pattern and are therefore easily recognised. CdLS is one of these. ${ }^{[3]}$

CdLS is a rare and clinically well-defined syndrome. Growth retardation, microcephaly, synophrys, long curved eyelashes, thin lips with facing-down convexity and a 
long filtrum are seen in all cases. ${ }^{[1,4]}$ Verma et al. ${ }^{[2]}$ reported in their series of 180 cases that all patients had microcephaly and pubertal delay, $97 \%$ had hirsutism, and $68 \%$ were of low birth weight. ${ }^{[2]}$ In our case, synophrys, a wide frenulum and a hirsute forehead were noted.

The cause of CdLS is unknown. A defect on chromosome 3q26.3 has been shown in cases with a family history or intermarriage..$^{[5]}$ In sporadic and familial cases, mutations of the NIPBL (nipped-Blike) gene, which is a cohesin regulator at the 5th chromosome, have been described. ${ }^{[6]}$ Bhuiyan et al. ${ }^{[7]}$ reported NIPBL mutation in $56 \%$ of 39 cases of CdLS. Our patient reported no intermarriage or family history.

Findings suggesting a diagnosis of CdLS in the prenatal period are increased nuchal translucency in the first trimester, symmetrical intrauterine developmental restriction (SIDR), significant defects in the upper extremities, and a dysmorphic facial appearance. ${ }^{[8-10]}$ Sekimoto et al. ${ }^{[9]}$ reported SIDR in 95\% of patients diagnosed with CdLS, skeletal anomalies in $81 \%$, facial dysmorphism in $50 \%$ and fetal diaphragmatic hernia in 50\%. Polyhydramnios was reported in 2 cases and nuchal translucency in 4 . A prenatal diagnosis could be made in 6 cases only ${ }^{[11-12]}$ Our patient also had a dysmorphic facial appearance and phocomelia on the right.

As in our case, because the findings on prenatal ultrasonography are nonspecific, CdLS can usually only be diagnosed after birth. As prenatal genetic diagnosis is possible and there is a risk that the syndrome will recur in subsequent pregnancies because of mosaicism, genetic counselling should be provided to the families of affected children. ${ }^{[13]}$

1. Weichert J, Schröer A, Beyer DA, Gillessen-Kaesbach G, Stefanova I. Cornelia de Lange syndrome: Antenatal diagnosis in two consecutive pregnancies due to rare gonadal mosaicism of NIPBL Antenatal dig mutation. J M

2. Verma L, Passi S, Gauba K. Brachman de Lange syndrome. Contemp Clin Dent 2010;1(4):268-270. [http://dx.doi.org/10.4103/0976-237X.76399]

3. Horsfield JA1, Print CG, Mönnich M. Diverse developmental disorders from the one ring: distinct molecular pathways underlie the cohesinopathies. Front Genet 2012;12(3):171. [http://dx.doi. $\operatorname{org} / 10.3389 /$ fgene.2012.00171]

4. Reddy HB, Neelaveni K, Hari Kumar KV. Cornelia de Lange syndrome. Indian J Endocrinol Metab 2013;17(4):763. [http://dx.doi.org/ 10.4103/2230-8210.113779]

5. Boyle MI, Jespersgaard C, Brøndum-Nielsen K, Bisgaard AM, Tümer Z. Cornelia de Lange syndrome. Clin Genet 2015;88(1):1-12. [http://dx.doi.org/10.1111/cge.12499]

6. Gillis LA, McCallum J, Kaur M, et al. NIPBL mutational analysis in 120 individuals with Cornelia de Lange syndrome and evaluation of genotype-phenotype correlations. Am J Hum Genet 2004;75(4):610-623. [http://dx.doi.org/10.1086/424698]

7. Bhuiyan ZA, Klein M, Hammond P, et al. Genotype-phenotype correlations of 39 patients with Cornelia de Lange syndrome: The Dutch experience. J Med Genet 2006;43(7):568-575. [http:// dx.doi.org/10.1136/jmg.2005.038240]

8. Boog G, Sagot F, Winer N, David A, Nomballais MF. Brachman-de Lange syndrome: A cause of early symmetric fetal growth delay. Eur J Obstet Gynecol Reprod Biol 1999;85(2):173-177. [http:// dx.doi.org/10.1016/S0301-2115(99)00021-4]

9. Sekimoto H, Osada H, Kimura H, Arai K, Sekiya S. Prenatal findings in Brachmann-de Lange syndrome. Arch Gynecol Obstet 2000;263(4):182-184. [http://dx.doi.org/10.1007/s004040050278]

10. Huang WH, Porto M. Abnormal first-trimester fetal nuchal translucency and Cornelia de Lange syndrome. Obstet Gynecol 2002;99(5):956-958. [http://dx.doi.org/10.1016/S0029-7844(02)01982-8]

11. Chong K, Keating S, Hurst S, et al. Cornelia de Lange syndrome (CdLS): Prenatal and autopsy findings. Prenat Diagn 2009;29(5):489-494. [http://dx.doi.org/10.1002/pd.2228]

12. Akahori Y, Masuyama H, Masumoto Y, Hiramatsu Y. Three-dimensional ultrasound findings in Cornelia de Lange syndrome: A case report. Case Rep Obstet Gynecol 2012;2012:568351. [http://dx.doi.org/10.1155/2012/568351]

13. Slavin TP, Lazebnik N, Clark DM, et al. Germline mosaicsm in Cornelia de Lange syndrome. Am J Med Genet A 2012;158A(6):1481-1485. [http://dx.doi.org/10.1002/ajmg.a.35381] 